\title{
Experimental Studies of the Coulomb Force Effects in Deuteron- Proton Break-up Reaction at Medium Energy Regime
}

\author{
I. Ciepał ${ }^{1}$, a , W. Parol ${ }^{2}$, N. Kalantar-Nayestanaki ${ }^{3}$, G. Khatri², St. Kistryn², B. Kłos ${ }^{4}$, A. Kozela ${ }^{1}$, \\ P. Kulessa ${ }^{1}$, J. Messchendorp ${ }^{3}$, I. Skwira-Chalot ${ }^{5}$, E. Stephan ${ }^{4}$, and B. Włoch ${ }^{6}$ \\ ${ }^{1}$ Institute of Nuclear Physics PAS, PL-31342, Kraków, Poland \\ ${ }^{2}$ Institute of Physics, Jagiellonian University, PL-30348, Kraków, Poland \\ ${ }^{3} \mathrm{KVI}-\mathrm{CART}$, University of Groningen, NL-9747 AA Groningen, The Netherlands \\ ${ }^{4}$ Institute of Physics, University of Silesia, PL-40007, Katowice, Poland \\ ${ }^{5}$ Faculty of Physics, University of Warsaw, PL-02093, Warsaw, Poland \\ ${ }^{6}$ AGH - University of Science and Technology, Faculty of Physics and Applied Computer Science, \\ al. A. Mickiewicza 30, PL-30059 Kraków, Poland
}

\begin{abstract}
A set of differential cross-section data of the ${ }^{1} \mathrm{H}(d, p p) n$ breakup reaction at 130 and $160 \mathrm{MeV}$ deuteron beam energies has been measured in the forward polar angles domain. The data were collected with the use of the Germanium Wall (FZ Jülich) and BINA (KVI Groningen) detectors. This part of the phase-space is special with respect to the dominant Coulomb force influence on the system dynamics. The data are compared with the theoretical calculations based on the Argonne V18 potential supplemented with the long-range electromagnetic component. The predictions also include the Urbana IX three nucleon force model. The strongest Coulomb effects are found in regions where the relative energy of the two protons is the smallest.
\end{abstract}

\section{Introduction}

Deuteron break-up in collision with one proton, leading to a final state of three-nucleon $(3 \mathrm{~N})$ continuum is one the simplest systems to study, providing a good testing ground for the nuclear Hamiltonian. Such a process offers a rich kinematics which makes it more selective regarding the interaction. One of the most extensively studied $3 \mathrm{~N}$ observables is the differential cross section, very sensitive to different pieces of the system dynamics like three-nucleon force (3NF), Coulomb interaction or relativistic effects. One can investigate their influence on the observables at various parts of the phase space, simply by choosing different geometries of the outgoing nucleons.

In the very forward angular range, investigated with the Germanium Wall and BINA detectors (see Sec. 2), the dynamics is dominated with the Coulomb force influence. For the first time, such significant effects were observed in the $d p$ breakup differential cross-section data measured at 130 $\mathrm{MeV}$ [1]. Then, the Coulomb force effects were studied in more details in a dedicated experiment at FZJ [2-4].

The first calculations which included the Coulomb interaction were done with CD Bonn and CD

\footnotetext{
a e-mail: izabela.ciepal@ifj.edu.pl
} 
Bonn $+\Delta$ potentials $[5,6]$ for elastic proton-deuteron scattering. Afterwards, they were developed for the $p d$ breakup process [7] and the existing experimental data [1] strongly supported the significant effects predicted by the calculations. The dedicated experiment provided the possibility to test them at the new level of accuracy [4]. Recently, a consistent theoretical treatment of a phenomenological 3NF (Urbana IX) and the Coulomb force has been achieved also for the Argonne V18 (AV18) potential [8] that allows to investigate the role of both effects to a high level of accuracy.

In the present work the extensive data sets obtained in a series of the experiments are used to investigate the Coulomb force influence on the differential cross section.

\section{Experiments}

The experiments at FZJ was conducted with the Germanium Wall (GeWall) setup [4] at the deuteron beam energy of $130 \mathrm{MeV}$. GeWall consisted of three high-purity germanium position sensitive detectors. Two different types of the detectors were used: a thin transmission detector Quirl with an excellent spatial resolution for determining the position and energy loss ( $\Delta E$ detector) of the passing charged particles, and two thick detectors $E 1$ and $E 2$ for measurement of particle energies with an excellent resolution. The angular acceptance of the apparatus was $5^{\circ}-14^{\circ}$ for the polar and $2 \pi$ for the azimuthal angles.

With the BINA detector and the deuteron beam energy of $160 \mathrm{MeV}$ the new rich contribution to the database of the differential cross section was obtained $[9,10]$. The apparatus is composed of the two main parts called Wall and Ball. Wall, covering the angular range from $15^{\circ}$ to $37^{\circ}$, is built of the MWPC for momenta reconstruction, $\Delta E$ (24 strips) and $E$ (10 slabs) scintillator detectors. The $\Delta E$ and $E$ create virtual matrix of 120 telescopes. The backward part is ball-shaped and consists of 149 phoswich detectors which cover polar angles between $40^{\circ}$ and $160^{\circ}$. The Ball plays two roles: of the particle detector and scattering chamber.

\section{Studies of Coulomb force effects}

In the investigated part of the phase space, the electromagnetic interaction plays a crucial role. Therefore, calculations which take into account both dynamical components, i.e., the $3 \mathrm{NF}$ and the Coulomb force, are able to properly reproduce the data. Adding the electromagnetic force into the calculations does not essentially change the quality of the data description at large values of the invariant mass of the protons emerging from the breakup reaction $\sqrt{s_{p p}}$. However, at small $\sqrt{s_{p p}}$ values the observed discrepancies are almost totaly removed $[1,4]$. The inclusion of $3 \mathrm{NF}$ and Coulomb effects into the calculations does not remove completely the disagreement between data and theoretical predictions. There are still some discrepancies at large $\sqrt{s_{p p}}$ [3] which can be interpreted as indication that parts of the dynamics are missing, either relativistic effects or unresolved problems in our understanding of the $3 \mathrm{NF}$ structure.

The experimental investigations at FZJ [4] were focusing on a very forward and narrow part of the phase space where the predicted Coulomb force effects are significant. Very recently also a new set of the breakup cross section at $160 \mathrm{MeV}$ was obtained $[9,10]$, allowing one to search for the effects but at slightly higher energy. The new data were evaluated for angular configurations on a grid defined by the polar angles of the two protons $\theta_{1}, \theta_{2}$ given in a range of $17^{\circ}-38^{\circ}$ with a step of $2^{\circ}$, and their relative azimuthal angle $\varphi_{12}\left(20^{\circ}-180^{\circ}\right)$, with a step of $20^{\circ}$. Sample preliminary distributions of the differential cross section are presented in Fig. 1. The importance of the Coulomb interaction for correct description of the data at low $\varphi_{12}$ (left panel) is quite clear.

To check in a systematic way how the electromagnetic processes influence the data, the cross sec- 

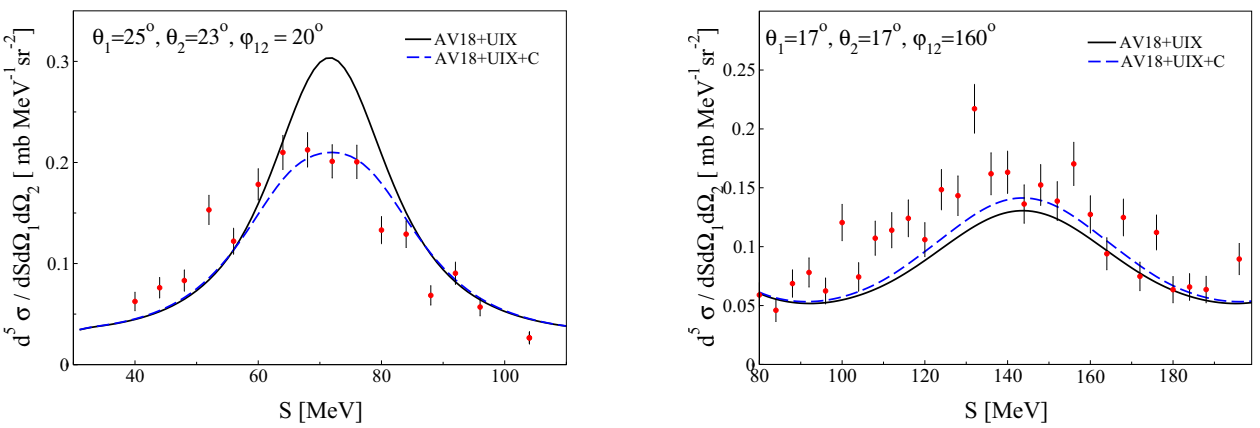

Figure 1. (Color online) Differential cross-section data for the $d p$ breakup reaction at $160 \mathrm{MeV}$ at two different kinematical configurations (specified in the panels). In the configuration presented on the left panel significant Coulomb force effects are observed. The data are compared to the AV18-based calculations and are described in the legend.

tions were integrated over $S$ and studied as a function of the $\varphi_{12}$ variable. The same integration, limited by the experimental energy threshold of $30 \mathrm{MeV}$, was done for the model predictions of AV18+UIX and AV18+UIX+C. The two closest in $\theta$-distance geometries originating from the 130 and $160 \mathrm{MeV}$ datasets were investigated, see Fig. 2.

The calculations which neglect the Coulomb force usually overestimate the data at the lowest relative azimuthal angle $\left(\varphi_{12}<40^{\circ}\right)$ for both the studied datasets. Such a behavior is consistent with the intuitive understanding (neglecting any interference of the dynamical ingredients) - the Coulomb repulsion decreases the number of protons in configurations with small relative energies. This corresponds to a situation when the protons stay close together for a relatively long time and the repulsion can lead to a significant change of their initial trajectories. For higher $\varphi_{12}$, one observes opposite situation at $130 \mathrm{MeV}$, whereas at $160 \mathrm{MeV}$, only very small Coulomb influence is seen.

With growing $\varphi_{12}$ the discrepancy changes sign and at a certain point the theoretical curves intersect. Such points are visible at $\varphi_{12}=60^{\circ}$ and $\varphi_{12}=40^{\circ}$ at 130 and $160 \mathrm{MeV}$, respectively. This refers
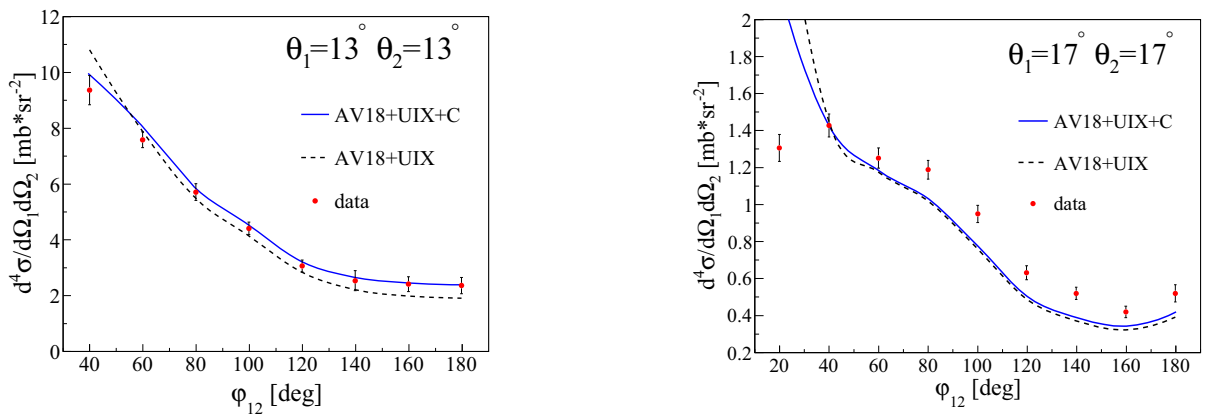

Figure 2. Differential, integrated over energy, cross-section values presented as a function of the relative azimuthal angle $\varphi_{12}$, for a given $\theta_{1}, \theta_{2}$ combination. The presented data were obtained at $130 \mathrm{MeV}$ (left panel) and at $160 \mathrm{MeV}$ (right panel) deuteron beam energies. The data are compared with the calculations indicated in the legend.

to a situation when the number of "incoming" and "outgoing" protons is balanced and the net effect 
of the Coulomb force is equal to zero. In a range of the higher relative angles the number of protons is increased. This, in turn, is caused by the protons originating from the region where the repulsion is strong.

The results obtained at $130 \mathrm{MeV}$ revealed a quite strong influence of the Coulomb force effects on the breakup reaction. The quantitative comparisons with the available predictions for the $3 \mathrm{~N}$ systems demonstrates a crucial role of the electromagnetic component in the data description [4]. The data evaluated at $160 \mathrm{MeV}$ are still preliminary and one also needs to estimate the possible systematic effects to draw final conclusions on the quality of description provided by the calculations.

\section{Summary and conclusions}

Precise and systematic studies of the breakup reaction in a large part of the phase space are very important for understanding of the interaction between nucleons in few-nucleon systems. Currently available theoretical approaches which try to model the interaction need very precise and large experimental database to be verified and further developed. Within these predictions different pieces of the dynamics can be studied separately and also their mutual interplay can be investigated. Moreover, there is still strong need to have possibly complete theoretical treatments including all ingredients of the $3 \mathrm{~N}$ system dynamics (3NF, Coulomb interaction, relativistic effects).

New experiments to study $3 \mathrm{~N}$ system dynamics are planned, including also investigations of the three-body system in the four-body environment. The data obtained with the WASA detector at FZJ at higher energies are under analysis. They will allow one to unambiguously fix a relevance of the 3NF. A new scientific program concentrated on investigation of the few-nucleon systems dynamics was proposed to be carried out with the use of the BINA detector at the Cyclotron Center Bronowice (CCB) in Cracow. Moreover, an experimental apparatus which will be used in measurements with a polarized ${ }^{3} \mathrm{He}$ target is under construction. Such studies planned at CCB will allow one to fill a data-deficient sector of $4 \mathrm{~N}$ systems.

\section{Acknowledgements}

This work was partially supported by the Polish National Science Center under Grants No. 2012/05/E/ST2/02313 (2013-2016) and No.DEC-2013/10/M/ST2/00420.

\section{References}

[1] St. Kistryn et al., Phys. Lett. B 641, 23-27 (2006)

[2] I. Ciepał et al., EPJ Web of Conferences 37, 09011 (2012)

[3] I. Ciepał et al., Few-Body Syst. 601, 1-5 (2013)

[4] I. Ciepał et al., Few-Body Syst., http://dx.doi.org/10.1007/s00601-015-1014-8, 1-26 (2015) doi=10.1007/s00601-015-1014-8

[5] A. Deltuva, A.C. Fonseca, P.U. Sauer, Phys. Rev. C 71, 054005 (2005)

[6] A. Deltuva, A.C. Fonseca, P.U. Sauer, Phys. Rev. C 72, 054004 (2005)

[7] A. Deltuva, A.C. Fonseca, P.U. Sauer, Phys. Rev. C 73, 057001 (2006)

[8] A. Deltuva, Phys. Rev. C 80, 064002 (2009)

[9] W. Parol et al., Acta Physica Polonica B, Vol. 45, No. 2, 527-532 (2013)

[10] W. Parol et al., EPJ Web of Conferences 81, 06007 (2014) 